\title{
Hard Diffractive Results and Prospects at the Tevatron ${ }^{1}$
}

\author{
Krisztian Peters \\ Department of Physics \& Astronomy, University of Manchester, \\ Manchester M13 9PL, UK \\ on behalf of the DØ and CDF Collaborations
}

\begin{abstract}
We review hard diffractive results and prospects at the Tevatron with an emphasis on factorization breaking in diffractive processes. Upper limits on the exclusive di-jet and $\chi_{c}^{0}$ production cross sections at CDF and the status of the DØ Forward Proton Detectors are discussed.
\end{abstract}

\section{DIFFRACTION AT THE TEVATRON}

Diffractive events are mediated by the exchange of color singlets with vacuum quantum numbers and have clear experimental signatures. These are on one hand rapidity gaps: the absence of particles in some regions of rapidity in contrast to non-diffractive events where gaps are filled by additional soft parton interactions which yields an exponential suppression of rapidity gaps. On the other hand tagged protons or anti-protons: $p$ or $\bar{p}$ scattered at small angle and measured in Roman Pots far away from the interaction point. Depending on these rapidity gaps and tags, the main diffractive event topologies at the Tevatron are: single diffraction (SD), double diffraction (DD) or double Pomeron exchange (DPE). Single diffraction is characterized by a leading proton or anti-proton which escapes the collision intact and the presence of a further particle or a di-jet resulting from a hard scattering separated by a rapidity gap. Double diffraction is characterized by a gap in the central region and dissociated protons and anti-protons. In DPE there is a gap on both, the proton and anti-proton side, with a central produced di-jet or other particles. Proton and anti-proton remains intact.

In RUN II the CDF Forward Proton Detectors (FPDs) include Roman Pot detectors approximately $57 \mathrm{~m}$ from the interaction point. These consist of three stations and each station comprises one scintillation fiber detector and one trigger counter. Beam Shower Counters, a set of scintillation counters around the beam pipe, are used to reject nondiffractive (ND) background at the trigger level. This makes it possible to collect diffractive data at high luminosities. The energy flow in the event in the very forward direction is measured by Miniplug Calorimeters. These consist of alternating layers of lead plates and liquid scintillator readout. It has a towerless geometry without dead regions due to

\footnotetext{
${ }^{1}$ Presented at the XXXV International Symposium on Multiparticle Dynamics 2005, August 9-15, 2005, Kromeriz, Czech Republic
} 
(a)

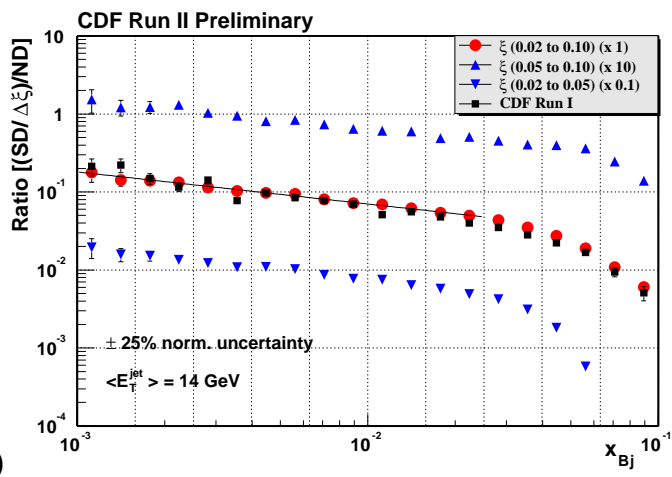

(b)

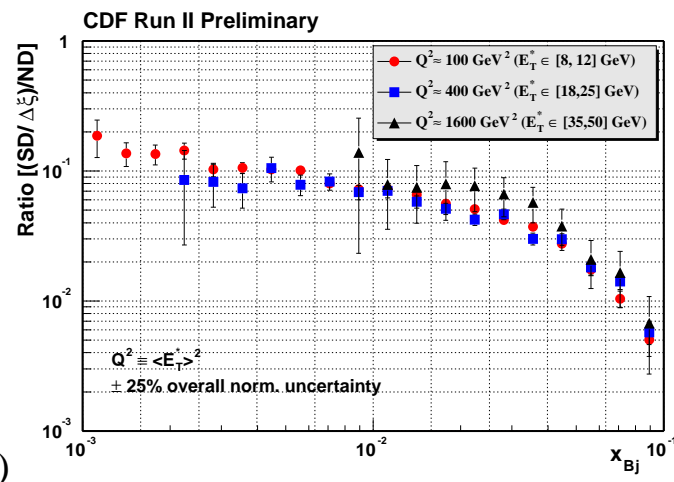

FIGURE 1. Ratio of diffractive to non-diffractive di-jet event rates as a function of $x_{B_{j}}$ at CDF for different $\xi$ ranges and compared to Run I (a) and for different $Q^{2}$ values.

the lack of internal mechanical boundaries. The DØ FPDs are described in the following.

\section{FACTORIZATION IN DIFFRACTION}

One of the central issues of diffraction is whether hard diffractive processes obey QCD factorization. As Collins proved [1] for the general class of diffractive DIS processes, the cross section can be described as the convolution of a hard scattering matrix element (process dependent) and parton density functions (process independent). The question arises if this factorization theorem is more general, are parton densities really universal in diffractive exchange? Can we use them for different collider processes and energies? To answer this question is fundamental for the understanding of diffraction and it is also important to extrapolate Tevatron results to the LHC. The general strategy to prove factorization is to extract parton density functions (PDFs) and compare predictions to measurements of other processes and experiments.

Before the extraction of PDFs, diffractive fractions already yield some insight. Both, $\mathrm{CDF}$ and $\mathrm{D} \varnothing$, measured for different processes the fractions of events with one gap to all events. It was found that all ratios are at the order of $1 \%$ at the Tevatron which would support a more general factorization theorem. However the ratio of $1 \%$ yields an uniform gap suppression w.r.t. HERA where the diffractive rates are approximately 10 times higher. This discrepancy indicates already the breakdown of QCD factorization.

CDF measured in Run I the diffractive structure function of the anti-proton from SD di-jets and the result was compared with expectations from diffractive DIS measurements of H1 [2]. These events can be described in terms of a Pomeron emitted from the anti-proton and scattering with a parton from the proton. The diffractive structure function was obtained by measuring the ratio of the diffractive and non-diffractive cross section. The product of this ratio with the known non-diffractive structure function gives the result on the diffractive structure function. Although the shapes of the structure functions from HERA and Tevatron have similar shapes, there is a normalization discrepancy of a factor of 10. This result again confirms the breakdown of QCD factorization between the Tevatron and HERA as already expected from the result on the diffractive fractions. 


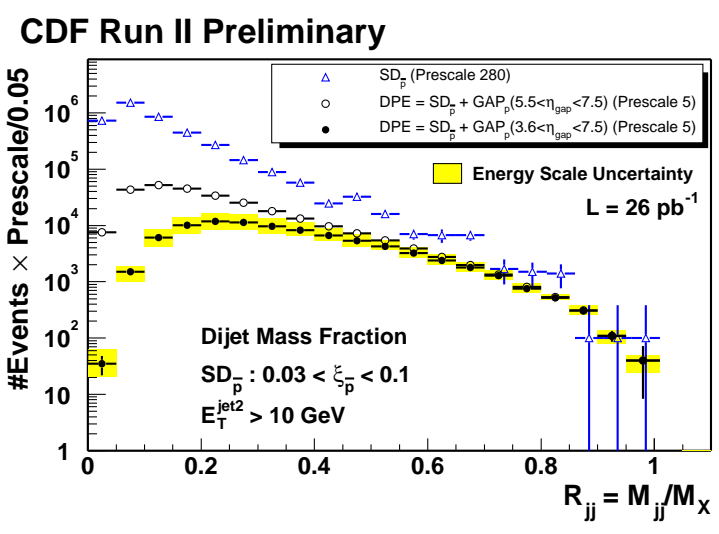

(a)

FIGURE 2. Di-jet mass fraction for different rapidity gap selections (a) and di-muon plus photon invariant mass in the exclusive sample compared to Monte Carlo predictions (b) at CDF.

One possibility to explain these observations is that, since there are more spectator partons in $p \bar{p}$ collisions w.r.t. $\gamma^{*} p$ collisions, the rate of gap destructions due to soft partonic interactions is larger at the Tevatron. One attempt to describe this rate is made with the introduction of the concept of the "gap survival probability" $|S|^{2}[3,4]$. The observation that the shapes of the two structure functions from HERA and the Tevatron are similar supports this concept. The gap survival probability factor corrects the normalization discrepancy.

In Run II the diffractive di-jet sample was collected with a dedicated trigger which selects events with at least one calorimeter tower above the $5 \mathrm{GeV} E_{\mathrm{T}}$ threshold and a threefold Roman Pot Spectrometer coincidence. Calorimeter information is used to determine the momentum loss of the anti-proton,

$$
\xi_{\bar{p}}=\frac{\sum E_{T} e^{-\eta}}{\sqrt{s}}
$$

SD and background regions are selected according to the measured $\xi_{\bar{p}}$ values. A large number of events are at $\xi_{\bar{p}} \sim 1$ which are due to the overlap of at least one ND contribution. A plateau is observed in the $\xi_{\bar{p}}$ distribution which results from a distribution proportional to $1 / \xi_{\bar{p}}$ as expected for diffractive production.

In Fig. 1 the ratio of SD to ND event rates is plotted versus Bjorken $x_{B_{j}}$. In Fig. 11a) this ratio is integrated over three different $\xi$ regions and compared to the Run I result. There is no $\xi$ dependence observable between 0.03 and 0.1 . Furthermore the slope and normalization agrees and thus confirms the Run I result. In Fig. 1(b) the same ratio is plotted for three different $Q^{2}$ values, where $Q^{2}$ is the mean jet transverse energy $\left\langle\left(E_{\mathrm{T}}^{1}+E_{\mathrm{T}}^{2}\right) / 2\right\rangle^{2}$. There is no appreciable $Q^{2}$ dependence in the observable region of $100<Q^{2}<1600 \mathrm{GeV}^{2}$. Thus both structure functions seem to have a similar $Q^{2}$ evolution which does not disfavor any of the two mechanisms of hard diffraction, namely the existence of a hard Pomeron (exchange of a colorless object) or a soft color 
rearrangement in the final state.

Using the di-jet sample CDF extracted also DPE di-jet production events. These events are characterized by a leading anti-proton, two jets in the central pseudorapidity region and a large rapidity gap on the outgoing proton side. If factorization holds the ratio of DPE to SD, $R_{S D}^{D P E}\left(x_{p}, \xi_{p}\right)$ has to be equal to the ratio of SD to ND, $R_{N D}^{S D}\left(x_{\bar{p}}, \xi_{\bar{p}}\right)$ (in LO QCD) for a fixed $x_{B_{j}}$ and $\xi$ value. Although the collected events have different $\xi$ ranges for the proton and anti-proton, the weighted average of $R_{N D}^{S D}\left(x_{\bar{p}}, \xi_{\bar{p}}\right)$ is flat in $\xi$ and the ratio was extrapolated to $\xi=0.02$. At this $\xi$ values the above mentioned ratios differ by a factor of 5 [5]. The deviation from unity yields again a breakdown of factorization. Since the formation of a second gap is less suppressed this result is coherent with the concept of the gap survival probability. The number of spectator partons does not changed with the formation of a second gap, i.e. one does not have to pay the price for the gap two times.

In the same manner as was previously done by the SD/ND ratio, the diffractive structure function can be extracted from the DPE/SD ratio. The obtained result now approximately agrees with expectations from $\mathrm{H} 1$ leading again to the above mentioned conclusions.

\section{SEARCH FOR EXCLUSIVE EVENTS}

Since the CDF Roman Pots have been installed at the end of Run I, CDF collected in Run II two orders of magnitude more di-jet data which made a study of exclusive di-jet production in DPE possible. The strategy was to obtain an inclusive DPE di-jet event sample and look for exclusive signature using the di-jet mass fraction $R_{j j}=M_{j j} / M_{X}$ where the di-jet mass is divided by the mass of the rest of the system excluding the proton and anti-proton. In Fig. 2 (a) the obtained number of events is plotted versus the di-jet mass fraction where no gap, a narrow gap and a wide gap was required on the proton side. The result is a smoothly falling spectrum all the way down to $R_{j j}=1$ and a similar event yield at high mass fraction regardless of the gap requirements. From this it follows that no significant excess due to exclusive di-jets is seen at high $R_{j j}$. An upper limit on the exclusive di-jet production cross section is calculated based on events with $R_{j j}>0.8$. For example requiring a minimum jet transverse energy of $10 \mathrm{GeV}$, this upper limit is

$$
E_{\mathrm{T}}^{\min }=10 \mathrm{GeV}: \quad \sigma\left(R_{j j}>0.8\right)<1.1 \pm 0.1(\text { stat }) \pm 0.5(\text { sys }) \mathrm{nb} .
$$

There are also other production channels available in DPE, we mention here the exclusive $\chi_{c}^{0}$ production. This process is of particular interest, since the $\chi_{c}^{0}$ quantum numbers are very similar to the ones of the Higgs boson, thus it can be used to test and normalize the predictions for exclusive Higgs production at the LHC. CDF did an analysis in Run II where the $\chi_{c}^{0}$ further decays in a $J / \psi+\gamma .93 \mathrm{pb}^{-1}$ of di-muon triggered data was used and events have been selected in the $J / \psi$ mass window. A large rapidity gap on both the proton and anti-proton side was required. 10 events have been found which are exclusive $\chi_{c}^{0}(\rightarrow J / \psi+\gamma)$ candidates. In Fig. 2 2 b) the di-muon plus photon invariant mass of the 10 events is compared with a sample of generated $\chi_{c}^{0}$ 


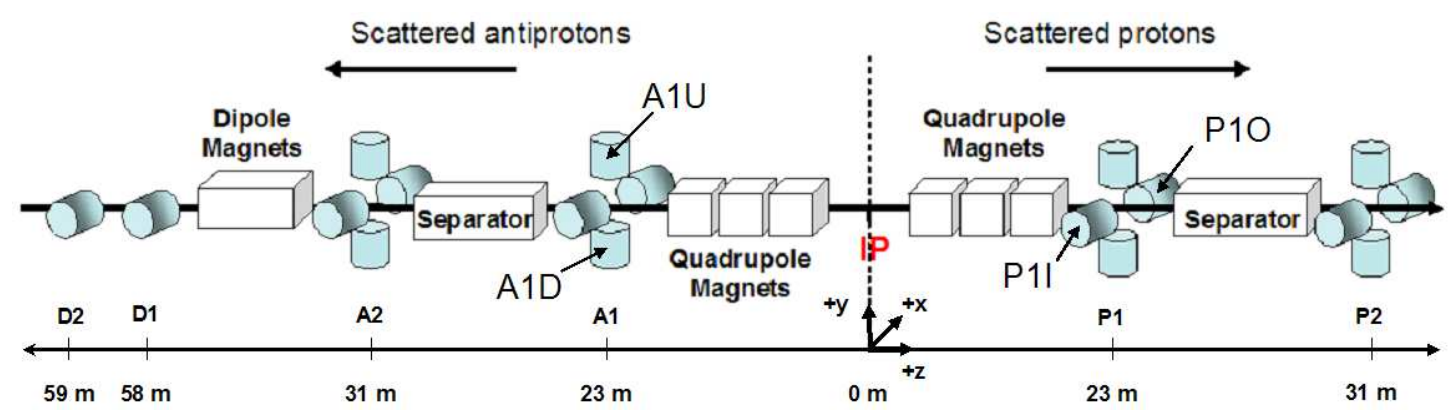

FIGURE 3. The Forward Proton Detector at D $\varnothing$. Quadrupole Pots are named P or A when placed on the proton or the anti-proton side, respectively. Dipole Pots are named D.

events passed through a detector simulation. The invariant mass is consistent with that of the $\chi_{c}^{0}$, although the mean mass is higher and the distribution broader in the data than in the simulation. This may be due to the simulation or there may be contributions from cosmic events, higher mass $\chi_{c}$ mesons or $J / \psi+\pi^{0}$ events. Since it is very difficult to fully understand the background one may calculate an "upper limit" on exclusive $\chi_{c}^{0}$ production assuming that the observed 10 events are all $J / \psi+\gamma$ events. This upper limit is:

$$
\sigma=49 \pm 18(\text { stat }) \pm 39(\text { sys }) \mathrm{pb} .
$$

\section{DØ FORWARD PROTON DETECTORS}

The DØ Forward Proton Spectrometers have been installed and recently commissioned. These are 9 momentum spectrometers with 2 scintillating fiber detectors each, as schematically shown in Fig. 3. There is a dipole spectrometer located on the scattered anti-proton side behind the dipole magnets approximately $58 \mathrm{~m}$ away from the interaction point. They have in the range of $|t| \approx 0-1 \mathrm{GeV}^{2}$ and $\xi \approx 0.03-0.07$ a very good acceptance. Eight quadrupole spectrometers are on both side of the main detector approximately 23 and $31 \mathrm{~m}$ away from the interaction point, behind the quadrupole magnets and the separators. They have a very good acceptance in the region of: $|t| \approx 0.8-3.0$ $\mathrm{GeV}^{2}$ and $\xi \approx 10^{-3}-0.05$. The position detectors housed inside Roman Pots operate millimeters away from the beam, however outside of the ultra high vacuum. They enable the reconstruction of the high energy protons and anti-protons directly, thus providing a first time possibility of tagging both the protons and anti-protons and measuring their $\xi$ and $t$ dependence at the Tevatron.

The $\mathrm{D} \varnothing$ scintillating fiber detectors have 6 layers of scintillating fiber channels and one trigger scintillator layer. The fibers are oriented within $\pm 45^{\circ}$ to reconstruct hits and obtain redundancy. Furthermore every second channel is offset by $2 / 3$ fiber for a finer hit resolution. All 18 detectors regularly brought close to the beamline and diffractive samples being collected.

In a small dedicated test run of the FPDs in a stand alone mode the slope of the elastic cross section of proton anti-proton scattering was measured. In Fig. 4 the result (not normalized) is plotted and compared with theory predictions [6]. DØ access a new 


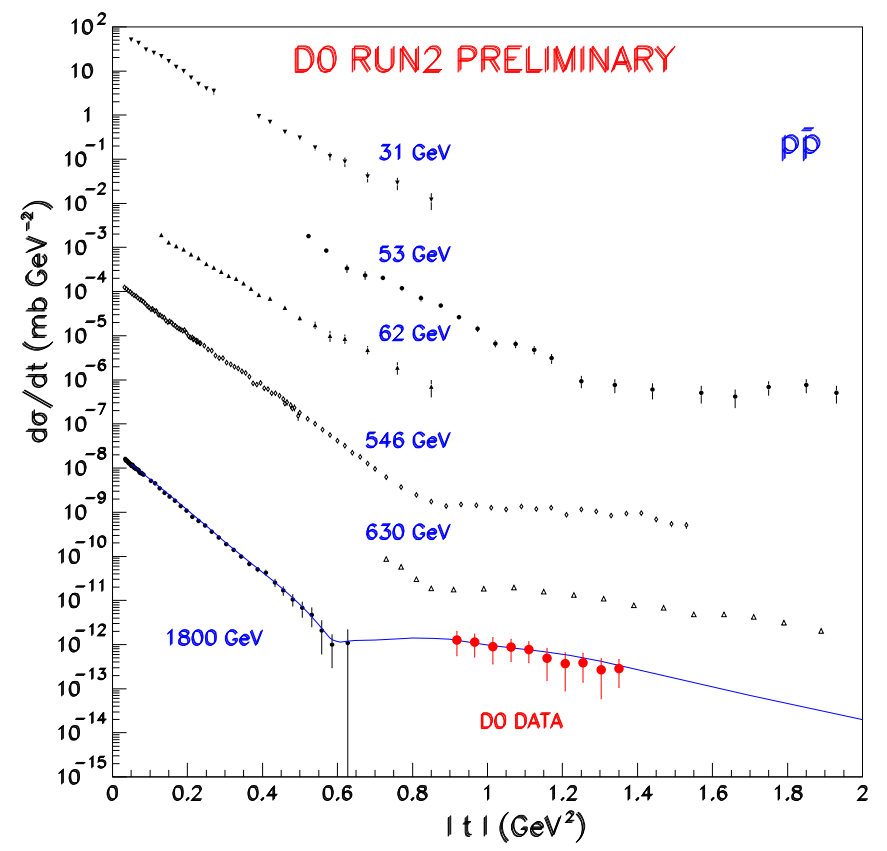

FIGURE 4. The slope of the elastic $p \bar{p}$ cross section measured at $\mathrm{D} \emptyset$ and compared to predictions of [6] (solid line).

kinematic domain with these measurements and the result of the slope agrees well with the model of Bock et al. [6]. This measurement is being redone using the fully integrated FPDs. The alignment and detector understanding of the Roman Pot detectors is in progress and more physics results are expected soon.

\section{REFERENCES}

1. J. C. Collins, Phys. Rev. D57, 3051-3056 (1998), hep-ph/9709499

2. T. Affolder, et al., Phys. Rev. Lett. 84, 5043-5048 (2000).

3. J. D. Bjorken, Phys. Rev. D47, 101-113 (1993).

4. E. Gotsman, E. M. Levin, and U. Maor, Phys. Lett. B309, 199-204 (1993), hep-ph/9302248

5. T. Affolder, et al., Phys. Rev. Lett. 85, 4215-4220 (2000).

6. M. Block, R. Fletcher, F. Halzen, B. Margolis, and P. Valin, Phys. Rev. D41, 978 (1990). 